\title{
Re: Quantification of Fractal Dimension and Shannon's Entropy in Histological Diagnosis of Prostate Cancer
}

\author{
de Arruda PF, Gatti M, Facio FN Jr, de Arruda JG, Moreira RD, Murta LO Jr, de Arruda LF, \\ de Godoy MFx
}

Faculdade de Medicina de São José do Rio Preto (FAMERP), SP, Brazil

BMC Clin Pathol 2013;18;13:6. doi: 10.1186/1472-6890-13-6.

\section{EDITORIAL COMMENT}

Fractal is a natural phenomenon or a mathematical set that exhibits a repeating pattern that displays at every scale. The new terms entropy and fractal are used for histopathological diagnosis of tumours. In this study, the aim was to quantify the fractal dimension and Shannon's entropy in the histological diagnosis of prostate cancer. After radical prostatectomy $(n=34)$, histological slides of normal, hyperplastic and tumor areas of the prostate were analyzed. Fractal dimension, Shannon's entropy and number of cell nuclei in these areas were compared. As Shannon's entropy quantifies the degree of complexity in information in histological slides, there is a high probability of differentiating tumor tissue from normal tissue, indicating that this method could be useful in the diagnosis of prostate cancer (Yogesan et al., 1996). They reported that fractal dimension analysis was sufficient to distinguish between tumoral and normal tissue in prostate at magnification at 40X and 100X. On the other hand, in the calculation of Shannon's entropy, magnification at 100X and 400X differentiated tumor tissue from both normal and hyperplastic tissue. In this study, the authors suggested that the quantification of the fractal dimension and Shannon's entropy, together with the number of cell nuclei, has potential clinical applications in the histological diagnosis of prostate cancer.

Fehmi Narter MD

\section{Re: Immunochromatographic Diagnostic Test Analysis Using Google Glass}

\section{Feng S, Caire R, Cortazar B, Turan M, Wong A, Özcan A.}

1 Electrical Engineering Department, ₹Bioengineering Department, §California NanoSystems Institute, and Department of Surgery,

David Geffen School of Medicine, University of California , Los Angeles, California, United States.

ACS Nano 2014;8:3069-3079.

\section{EDITORIAL COMMENT}

'Google Glass' can be pioneer in a new technology era. Google Glass will be used in many different technological fields in the future. In the near future, it is going to be used for diagnostic or therapeutic purposes in medicine. Recently, it is tested for these reasons in a few studies. In one of them, Feng and et al. demonstrated a Google Glass-based rapid diagnostic test (RDT) reader platform capable of qualitative and quantitative measurements of various lateral flow immunochromatographic assays and similar biomedical diagnostics tests. They tested this Google Glass-based diagnostic platform using qualitative human immunodeficiency virus (HIV) and quantitative prostate-specific antigen (PSA) tests. For the quantitative RDTs, they measured activated tests at various concentrations ranging from 0 to $200 \mathrm{ng} / \mathrm{mL}$ for free and total PSA. This wearable RDT reader platform running on Google Glass combines a hands-free sensing and image capture interface with powerful servers. These servers run custom image processing codes. The reader can be useful for real-time spatiotemporal tracking of various diseases and personal medical conditions, providing a valuable tool for epidemiology and mobile health. In the near future, Google Glass technology will be candidate for diagnostic and therapeutic options, telemedicine applications, resident training, surgical training, patient communications, and procedure analysis as well as for faster access to information. 